\title{
Insecticidal Efficacy against Shoot and Fruit Borer, Leucinodes orbonalis Guene
}

\author{
Sundar Pal* \\ Department of Entomology, DBS College of Agricultur and Allied Science, Selaqui, \\ Dehradun, Uttrakhand-248001, India \\ *Corresponding author
}

\begin{abstract}
A B S T R A C T

\begin{tabular}{|l|}
\hline Ke y w o r d s \\
Efficacy, Insecticides, \\
Leucinodes orbonalis
\end{tabular}

\section{Introduction}

Brinjal (Solanum melongena L.) is very much palatable among widely grown vegetables in different parts of the world. Among the Solanaceous vegetables, brinjal, Solanum melongena Linn. is the most common, popular and principal vegetable crop grown in many geographical parts in India. The area under brinjal cultivation is estimated at 679.4 thousand ha. with total production of 12438.7 thousand tonns (HSD, DAC \& FW, 2014-15, http://www.mospi.gov.in/statistical-year-

book-India/2016/178). There is a vast scope to make brinjal cultivation more paying by improving the productivity. Brinjal fruit borer, Leucinodes orbonalis Guenee (Pyralidae: Lepidoptera) is found throughout the topics in

Asia and Africa and is a minor pest in America. It is monophagous and very important pest of brinjal. Attack of this pest causes considerable damage to brinjal crop each year, affecting the quality and yield of the crop. The Larvae of this pest cause 12$16 \%$ damage to shoots and $20-60 \%$ damage to fruits (Alam, 1970 and Maureal et al., 1982). The pest is very active during rainy and summer season and often causes more than 90\% damage in Bangladesh (Ali and Rahman, 1980 and Kalloo, 1988) and up to $95 \%$ in India (Naresh et al., 1986). Larvae feeding inside shoots result in wilting of young shoots. Presence of wilted shoots in a brinjal field is a symptom of damage by this pest. The damaged shoots ultimately wither and drop off. This reduces plant growth, which in turn,
\end{abstract}


reduces fruit number and size. New shoots can arise but this delays crop maturity and the newly formed shoots are also subjected to larval damage. Larval feeding in flowers is a relatively rare occurrence resulting in failure to form fruits from damaged flowers.

\section{Materials and Methods}

The experiment investigation was conducted on Agriculture Farm of DBS School of Agriculture and Allied Science, Selaqui, Deharadun, Uttrakhand, India during summer season 2017. The experiment was laid in Randomized Block Design with three replications and eight treatments included a control. The spraying was done after the population reaching its ETL. Spraying was done by using air compressing knapsack sprayer. The incidence of the borer on the shoot and the fruit were recorded from the five randomly selected plants. The assessment of the shoot damage was done by calculating the number of damaged shoots and total number of the healthy shoots observed from five randomly selected plants per plot and expressed in percentage. Fruit infestation was recorded on each plucking date at $7^{\text {th }}$ and $14^{\text {th }}$ day after each spraying. Brinjal plants transplanted from nursery to plots at plant to plant and row to row distance $30 \times 60 \mathrm{~cm}^{2}$. The percent fruit damage was total number of affected fruits from each plot. The total yield of the marketable fruits obtained from different treatments was calculated and converted by considering the additional cost (cost of insecticides and operational charges) and benefit (compared to untreated control) in the respective treatments.

$$
\begin{aligned}
& \text { Percent fruit infestation }=\frac{\text { No. of infested fruit }}{\text { Total no. of fruits }} \times 100 \\
& \text { Percent shoot infestation }=\frac{\text { No. of infested shoot }}{\text { Total no. of shoot }} \times 100
\end{aligned}
$$

\section{Results and Discussion}

\section{Shoot infestation in brinjal by Leucinodes orbonalis Guene.}

Data recorded on insecticidal efficacy against shoot infestation in brinjal that all treatments were non-significant followed by control. Data was significant before one day insecticide application. First observation was recorded at $7^{\text {th }}$ day after first spray of insecticide and the minimum shoot infestation per cent was recorded from emamectin benzoate treated plots $15.18 \pm 3.7$ followed by indoxacarb, spinosad, deltamethrin, cypermethrin, carbofuran and azadirectin where shoot infestation were 27.26 $\pm 7.1, \quad 28.40 \pm 7.0$, $30.10 \pm 7.5, \quad 38.52 \pm 9.4, \quad 44.46 \pm 9.8$ and $48.64 \pm 11.8$ per cent. The shoot infestation reduction over the control were in descending order as 79.22, 62.69, 61.13, 58.81, 47.28, 39.16 and 33.43 per cent from emamectin benzoate, indoxacarb, spinosad, deltamethrin, cypermethrin, carbofuran and azadirectin, respectively. Insecticidal efficacy was nonsignificant between emamectin benzoate, indoxacarb and spinosad. Ghosh et al., (2009) recorded as the effectiveness of neem products was less effective against Leucinodes orbonalis.

Observation recorded at $14^{\text {th }}$ day after application of first spray, all treatments were non-significant against control. The minimum shoot infestation per cent was 6.61 1.9 , recorded from emamectin benzoate with high reduction $(89.81 \%)$ over the control. The maximum shoot infestation was noticed for azadirectin treated plots where reduction over the control was $22.19 \%$ where Kaur et al., (2014) and Kaur et al., (2014) were reported that the emamectin benzoate was highly toxic against $L$. orbonalis for Amritsar and Malerkotla populations with LC50 values 0.49 and $0.061 \mathrm{ppm}$ respectively followed by chlorantaniliprole, indoxacarb, delfin and 
spinosad. Data was non-significant after second spray, from all treatments followed by control. The shoot infestation reduction over control was recorded in ascending order 24.91, 39.81, 57.40, 57.55, 66.84, 75.66 and 80.05 per cent from azadirectin, carbofuran, cypermethrin, deltamethrin, spinosad, indoxacarb and emamectin benzoate, respectively, at $7^{\text {th }}$ day after insecticides application. Data was recorded at $14^{\text {th }}$ days after chemical application on shoot infestation, where maximum shoot infested were noticed from carbofuran treated plots but it was nonsignificant with azadirectin (Table 1).

The minimum shoot infestation recorded from emamectin benzoate treated plot which was significantly different to others. Raini et al., (2016) tested bioefficacy of five insecticides against Leucinodes orbonalis, where deltamethrin proved most effective in reducing shoot damage $(60.40 \%)$ over control.

\section{Fruit infestation in brinjal by Leucinodes orbonalis Guene.}

First observation was recorded on fruit infestation at $7^{\text {th }}$ day after insecticides application, where fruit infestation show in ascending order, $5.23 \pm 1.0,9.27 \pm 0.8,9.41 \pm 1.4$, $10.60 \pm 0.8, \quad 10.93 \pm 2.0, \quad 14.97 \pm 2.2$ and $16.30 \pm 2.8$ per cent from emamectin benzoate, indoxacarb, spinosad, deltamethrin, cypermethrin, carbofuran and azadirectin, respectively, where reduction were noticed 84.24, 72.07, 71.64, 68.05, 67.07, 54.90 and 50.88 per cent, respectively (Table 2 ).

Data recorded at $14^{\text {th }}$ day after application of fruit infestation, where fruit infestation was reduction over control as in descending order $83.78,77.59,70.00,70.00,62.17,51.81$, and 51.57 per cent from emamectin benzoate, indoxacarb, spinosad, deltamethrin, cypermethrin, carbofuran and azadirectin, respectively. The maximum fruit infestation was recorded from azadirectin treated plots where $18.03 \pm 1.7$ per cent were infested. The result recorded on fruit infestation per cent as non-significant, among emamectin benzoate, indoxacarb and spinosad treated plots at $7^{\text {th }}$ day after second spray, where fruit infestation reduction over the control were as 83.78, 71.96 and 68.17 per cent, respectively. Fruit infestation per cent recorded as $3.45 \pm 0.1$, $6.90 \pm 0.1, \quad 9.20 \pm 1.2, \quad 12.65 \pm 1.2, \quad 13.72 \pm 1.7$, $16.14 \pm 1.4$ and $17.30 \pm 2.2$ from emamectin benzoate, indoxacarb, spinosad, deltamethrin, cypermethrin, carbofuran and azadirectin at $14^{\text {th }}$ day after application of insecticides.

The maximum (89.30\%) and minimum $(46.37 \%)$ fruit infestation reduction over the control was recorded from azadirectin and emamectin benzoate treated plots. Adiroubane (2008) recorded that spinosad was effective at fruiting stage in brinjal and maximum per cent reduction of fruit damage and it was on par with oxymatrine. Raini et al., (2016) tested bioefficacy of five insecticides against Leucinodes orbonalis, where deltamethrin proved most effective in reducing fruit damage, on number basis $(88.87 \%)$ over control.

\section{Yield and cost benefit ratio in brinjal}

The maximum production of brinjal was recorded from emamectin benzoate treated plots followed by indoxacarb, spinosad, deltamethrin, cypermethrin, carbofuran and azadirectin, where increased yield over the control were $17.82,11.93,10.67,6.82,5.28$, 4.00 and $3.17 \mathrm{q} / \mathrm{ha}$, respectively. The maximum cost benefit ratio was from emamectin benzoate where returned cost was 7.57 rupees after per rupee investment. The maximum net returns of ₹.39348.67 were obtained in emamectin benzoate treated plots. The yields among the treatment were significant. Indoxacarb and spinosad treatments were non-significantly different. 
Table.1 Efficacy of novel insecticide against brinjal shoots infestation by Leucinodes orbonalis

\begin{tabular}{|c|c|c|c|c|c|c|c|c|c|}
\hline \multirow[t]{3}{*}{ Treatments } & \multicolumn{9}{|c|}{ Per cent mean of shoot infestation } \\
\hline & \multirow{2}{*}{$\begin{array}{c}\text { Before } \\
\text { application }\end{array}$} & \multicolumn{4}{|c|}{$1^{\text {st }}$ spray } & \multicolumn{4}{|c|}{$2^{\text {nd }}$ spray } \\
\hline & & $7^{\text {th }}$ DAS & $\begin{array}{l}\text { Reduction } \\
\text { over control } \\
(\%)\end{array}$ & $14^{\text {th }}$ DAS & $\begin{array}{l}\text { Reduction } \\
\text { over control } \\
(\%)\end{array}$ & $7^{\text {th }}$ DAS & $\begin{array}{l}\text { Reducti } \\
\text { on over } \\
\text { control }\end{array}$ & $14^{\text {th }}$ DAS & $\begin{array}{l}\text { Reduction } \\
\text { over control } \\
(\%)\end{array}$ \\
\hline $\begin{array}{c}\text { Azadirectin 0.03EC @ } \\
5 \mathrm{ml} / \mathrm{L} \\
\end{array}$ & $50.15 \pm 4.1$ & $48.64 \pm 11.8$ & 33.43 & $43.88 \pm 4.4$ & 22.19 & $44.17 \pm 1.9$ & 24.91 & $33.36 \pm 1.3$ & 25.57 \\
\hline $\begin{array}{c}\text { Carbofuran } 3 G 1.0 \mathrm{~kg} \\
\text { a.i./ha }\end{array}$ & $49.07 \pm 4.5$ & $44.46 \pm 9.8$ & 39.16 & $42.20 \pm 4.4$ & 22.60 & $35.41 \pm 3.8$ & 39.81 & $35.66 \pm 1.3$ & 20.44 \\
\hline $\begin{array}{c}\text { Cypermethrin } 25 \text { EC @ } \\
0.05 \%\end{array}$ & $51.06 \pm 3.6$ & $38.52 \pm 94$ & 47.28 & $34.07 \pm 3.4$ & 47.50 & $25.06 \pm 4.5$ & 57.40 & $27.65 \pm 2.2$ & 38.32 \\
\hline $\begin{array}{c}\text { Deltamethrin 2.8EC @ } 14 \\
\text { g a.i/ ha }\end{array}$ & $50.24 \pm 4.7$ & $30.10 \pm 7.5$ & 58.81 & $28.97 \pm 2.2$ & 55.35 & $24.98 \pm 6.5$ & 57.55 & $20.63 \pm 1.5$ & 53.98 \\
\hline $\begin{array}{c}\text { Emamectin benzoate25 } \\
\text { WG@ 11g a.i./ha }\end{array}$ & $52.85 \pm 7.2$ & $15.18 \pm 3.7$ & 79.22 & $6.61 \pm 1.9$ & 89.81 & $11.74 \pm 0.5$ & 80.05 & $4.60 \pm 1.1$ & 89.74 \\
\hline $\begin{array}{c}\text { Indoxacarb 14.5 SC@75g } \\
\text { a.i./ha }\end{array}$ & $49.15 \pm 7.8$ & $27.26 \pm 7.1$ & 62.69 & $14.40 \pm 2.5$ & 77.80 & $14.32 \pm 1.4$ & 75.66 & $44.82 \pm 1.7$ & 82.04 \\
\hline $\begin{array}{c}\text { Spinosad 45SC @ 75g } \\
\text { a.i./ha }\end{array}$ & $49.15 \pm 4.1$ & $28.40 \pm 7.0$ & 61.13 & $19.26 \pm 2.4$ & 70.32 & $19.51 \pm 2.3$ & 66.84 & $8.05 \pm 1.1$ & 74.34 \\
\hline Control & $50.89 \pm 13.3$ & $73.07 \pm 18.8$ & - & $64.89 \pm 4.4$ & - & $58.83 \pm 4.4$ & - & $11.50 \pm 1.1$ & - \\
\hline CD & 1.98 & 7.64 & - & 9.62 & - & 4.89 & - & 8.67 & - \\
\hline
\end{tabular}


Table.2 Efficacy of novel insecticide against brinjal fruit infestation by, Leucinodes orbonalis

\begin{tabular}{|c|c|c|c|c|c|c|c|c|c|}
\hline \multirow[t]{3}{*}{ Treatments } & \multirow{3}{*}{$\begin{array}{c}\text { Before } \\
\text { application }\end{array}$} & \multicolumn{8}{|c|}{ Per cent mean of fruit infestation } \\
\hline & & \multicolumn{4}{|c|}{$1^{\text {st }}$ spray } & \multicolumn{4}{|c|}{$2^{\text {nd }}$ spray } \\
\hline & & $7^{\text {th }}$ DAS & $\begin{array}{l}\text { Reductio } \\
\text { n over } \\
\text { control }\end{array}$ & $14^{\text {th }}$ DAS & $\begin{array}{c}\text { Reduction } \\
\text { over } \\
\text { control }\end{array}$ & $7^{\text {th }}$ DAS & $\begin{array}{c}\text { Reduction } \\
\text { over } \\
\text { control }\end{array}$ & $14^{\text {th }}$ DAS & $\begin{array}{c}\text { Reductio } \\
\text { n over } \\
\text { control }\end{array}$ \\
\hline Azadirectin 0.03EC @ 5ml/ & $26.46 \pm 5.5$ & $16.30 \pm 2.8$ & 50.88 & $15.95 \pm 1.4$ & 51.57 & $18.03 \pm 1.7$ & 44.84 & $17.30 \pm 2.2$ & 46.37 \\
\hline Carbofuran 3G 1.0 kg a.i./ha & $27.52 \pm 1.9$ & $14.97 \pm 2.2$ & 54.90 & $15.87 \pm 0.8$ & 51.81 & $16.98 \pm 1.7$ & 48.04 & $16.14 \pm 1.4$ & 49.94 \\
\hline Cypermethrin 25 EC @ 0.05\% & $27.89 \pm 3.5$ & $10.93 \pm 2.0$ & 67.07 & $12.46 \pm 2.1$ & 62.17 & $14.32 \pm 1.3$ & 56.20 & $13.72 \pm 1.7$ & 57.44 \\
\hline Deltamethrin 2.8EC @ $14 \mathrm{~g}$ a.i/ ha & $25.80 \pm 3.1$ & $10.60 \pm 0.8$ & 68.05 & $10.23 \pm 1.6$ & 70.00 & $13.07 \pm 1.6$ & 60.01 & $12.65 \pm 1.2$ & 60.76 \\
\hline $\begin{array}{c}\text { Emamectin benzoate 25 WG@ 11g } \\
\text { a.i./ha } \\
\end{array}$ & $26.50 \pm 4.9$ & $05.23 \pm 1.0$ & 84.24 & $03.69 \pm 0.2$ & 88.80 & $05.30 \pm 1.5$ & 83.78 & $03.45 \pm 0.1$ & 89.30 \\
\hline Indoxacarb 14.5 SC@ 75g a.i./ha & $26.46 \pm 5.5$ & $09.27 \pm 0.8$ & 72.07 & $07.38 \pm 0.5$ & 77.59 & $09.16 \pm 1.5$ & 71.98 & $06.90 \pm 0.1$ & 78.60 \\
\hline Spinosad 45SC @ 75g a.i./ha & $25.11 \pm 6.3$ & $09.41 \pm 1.4$ & 71.64 & $09.88 \pm 1.5$ & 70.00 & $10.40 \pm 1.3$ & 68.17 & $09.20 \pm 1.2$ & 71.46 \\
\hline Control & $27.52 \pm 1.9$ & $33.18 \pm 1.3$ & - & $32.94 \pm 0.2$ & - & $32.69 \pm 2.6$ & - & $32.25 \pm 1.8$ & - \\
\hline CD & 1.03 & 3.08 & - & 3.10 & - & 5.22 & - & 2.87 & - \\
\hline
\end{tabular}

Table.3 Pooled cost benefit ratio of insecticides against brinjal shoot and fruit borer, Leucinodes orbonalis

\begin{tabular}{|c|c|c|c|c|c|c|c|}
\hline S. No. & Treatments & $\begin{array}{l}\text { Total cost } \\
(\mathrm{Rs} / \mathrm{ha})+*\end{array}$ & $\begin{array}{l}\text { Yield } \\
\text { (q/h.) }\end{array}$ & $\begin{array}{l}\text { Increase } \\
\text { yield }(q / h)\end{array}$ & $\begin{array}{c}\text { Value of } \\
\text { increase yield } \\
\text { (Rs.) }\end{array}$ & $\begin{array}{l}\text { Net } \\
\text { return } \\
\text { (Rs.) }\end{array}$ & CBR \\
\hline 1. & Azadirectin 0.03EC @ 5ml/l & 4865.68 & 16.85 & 3.17 & 7925.00 & 3059.32 & $1: 0.63$ \\
\hline 2. & Carbofuran3G @ $1.0 \mathrm{~kg}$ a.i./ha & 5996.85 & 17.68 & 4.00 & 10000.00 & 4364.75 & $1: 0.77$ \\
\hline 3. & Cypermethrin 25 EC @ 0.05\% & 5423.50 & 18.96 & 5.28 & 13200.00 & 7776.50 & $1: 1.43$ \\
\hline 4. & Deltamethrin 2.8EC @14 g a.i/ha & 5425.50 & 20.50 & 6.82 & 17050.00 & 11624.50 & $1: 2.14$ \\
\hline 5. & Emamectin benzoate25 WG@11g a.i./ha & 5201.33 & 31.50 & 17.82 & 44550.00 & 39348.67 & $1: 7.57$ \\
\hline 6. & Indoxacarb 14.5 SC@ 75g a.i./ha & 5531.11 & 25.61 & 11.93 & 29825.00 & 24293.89 & $1: 4.39$ \\
\hline 7. & Spinosad 45SC @ 75g a.i./ha & 5672.68 & 24.35 & 10.67 & 26675.00 & 21002.32 & $1: 3.70$ \\
\hline 8. & Control & - & 13.68 & & & & \\
\hline
\end{tabular}

*Labour charges + machine rent charges + other charges + insecticides cost. Marketing price of brinjal= Rs. 25.00/kg. 
The highest increased yield was recorded from emamectin benzoate $(17.82 \mathrm{q} / \mathrm{ha})$, followed by indoxacarb $(11.93 \mathrm{q} / \mathrm{h})$, spinosad (10.67q/ha), deltamethrin $\quad(6.82 \mathrm{q} / \mathrm{h})$, cypermethrin $(5.28 \mathrm{q} / \mathrm{h})$, carbofuran $(4.00 \mathrm{q} / \mathrm{h})$ and azadirectin $(3.17 \mathrm{q} / \mathrm{h})$. Raini et al., (2016) found the highest (1:8.7) cost to benefit ratio was recorded in deltamethrin followed by fenvalerate (1:8.5), cypermethrin (1:6.5), chlorpyriphos (1:4.5), Prempt (1:1.9), malathion (1:0.6) and Nimbecidine (1: -0.3$)$ (Table 3). The cost benefit ratio was most economically emamectin benzoate (1:7.57) followed by indoxacarb (1:4.39), spinosad (1:3.70), deltamethrin (1:2.14), cypermethrin (1:1.43), carbofuran (1:0.77 and azadirectin (1:0.63). Raini et al., (2016) founded that deltamethrin recorded the highest marketable fruit yield of $132.27 \mathrm{q} / \mathrm{ha}$ and lowest was found in case of Nimbecidine (33.53 q/ha) where Bhagwan and Kumar (2017) recorded that the highest yield noticed in Cypermethrin 25 EC (204.16 q/ha) followed by Spinosad 45 SC (197.22 q/ha).

\section{Acknowledgement}

I am highly thankful to the Chairman, DBS College of Agriculture and Allied Science, Selaqui, Dehradun, Uttrakhand for partially supported to carry out the research work. My thankfulness also goes to Principal and other staff members of the college, who provided insight and expertise that greatly assisted the research.

\section{References}

Adiroubane, D. Raghuraman, K. (2008). Plant products and microbial formulation in the management of brinjal shoot and fruit borer, Leucinodes orbonalis (Guenee.). Zoological Record Journal of Biopesticides; 1(2): 124-129.

Alam MZ. (1970). Insect pest of vegetables and their control in Bangladesh. Agril. Inf. Serv. Dacca. Bangladesh, 132.

Ali MI, Rahman MS. (1980). Field evaluation if wilt disease and shoot and fruit borer attack of different cultivators of brinjal. Bangladesh J Agric Sci.; 7(2):193-194.

Bhagwan, D. S. and A. Kumar (2017). Field efficacy of cypermethrin and certain biopesticides against brinjal shoot and fruit borer, (Leucinodes orbonalis Guenee) on Brinjal (Solanum melongena L.). Journal of Pharmacognosy and Phytochemistry; 6(4): 1930-1933.

Ghosh, Sunil Kr.; Senapati, S. K. (2009). Seasonal fluctuation in the population of Leucinodes orbonalis Guen. in the sub-himalayan region of West Bengal, India and its control on eggplant (Solanum melongena L.). Journals@Ovid Full Text Precision Agriculture. 10(5):443-449.

Kalloo. (1988). Solanacesous crops. In Vegetable Breeding Raton, Florida; 2:520-570.

Kaur, Jagdeep, Kang, BK. Singh, Balwinder (2014). Ase line data for insecticide resistance monitoring in brinjal shoot and fruit borer, Leucinodes orbonalis guenee. Zoological Record Bioscan; 9(4): 1395-1398.

Kaur, Jagdeep, Kang, BK. Singh, Balwinder (2014). Base line data for insecticide resistance monitoring in brinjal shoot and fruit borer, Leucinodes orbonalis guenee. Zoological Record Bioscan; 9(4): 1395-1398.

Maureal AM, Noriel LM, Esguerra NM. (1982). Life history and behavior of eggplant fruit borer. Annal. Trop. Res.; 4(3): 178.

Naresh JS, Malik VS, Balan JS, Khokhar KS. (1986). A new record of trathala sp, a larval endoparasite attacking brinjal fruit borer, Leucinodes orbonalis 
Guenee. Bull. Entomol. New Delhi; 27(1):74.

Raina J., G. S Yadav and S. S Sharma (2016). Bioefficacy of some insecticides against shoot and fruit borer, Leucinodes orbonalis Guenee on brinjal under Hisar agro-climatic conditions during kharif season. Journal of Applied and Natural Science: 8 (4):1801-1805.

\section{How to cite this article:}

Sundar Pal. 2018. Insecticidal Efficacy against Shoot and Fruit Borer, Leucinodes orbonalis Guene. Int.J.Curr.Microbiol.App.Sci. 7(04): 1599-1605. doi: https://doi.org/10.20546/ijcmas.2018.704.180 